\title{
An Iterative Algorithm for Mixed Equilibrium Problems and Variational Inclusions Approach to Variational Inequalities
}

\author{
Yeong-Cheng Liou \\ Department of Information Management, Cheng Shiu University, Kaohsiung 833, Taiwan \\ Correspondence should be addressed to Yeong-Cheng Liou, simplex_liou@hotmail.com \\ Received 13 September 2009; Revised 10 November 2009; Accepted 10 January 2010 \\ Academic Editor: Nanjing Huang
}

Copyright (C) 2010 Yeong-Cheng Liou. This is an open access article distributed under the Creative Commons Attribution License, which permits unrestricted use, distribution, and reproduction in any medium, provided the original work is properly cited.

We present an iterative algorithm for finding a common element $x^{*}$ of the set of solutions of a mixed equilibrium problem and the set of a variational inclusion in a real Hilbert space. Furthermore, we prove that the proposed iterative algorithms strongly converge to $x^{*}$ which solves some variational inequality.

\section{Introduction}

Let $C$ be a nonempty closed convex subset of a real Hilbert space $H$. Let $F: C \rightarrow H$ be a nonlinear mapping, let $\varphi: C \rightarrow R$ be a function, and let $\Theta$ be a bifunction of $C \times C$ into $R$. Now we consider the following mixed equilibrium problem:

Find $u \in C$ such that $\Theta(u, y)+\varphi(y)-\varphi(u)+\langle F u, y-u\rangle \geq 0, \quad \forall y \in C$.

The set of solution of problem (1.1) is denoted by EP.

If $F=0$, then the mixed equilibrium problem (1.1) becomes the following mixed equilibrium problem:

Find $u \in C$ such that $\Theta(u, y)+\varphi(y)-\varphi(u) \geq 0, \quad \forall y \in C$, 
which was considered by Ceng and Yao [1]. If $\varphi=0$, then the mixed equilibrium problem (1.1) becomes the following equilibrium problem:

$$
\text { Find } u \in C \text { such that } \Theta(u, y)+\langle F u, y-u\rangle \geq 0, \quad \forall y \in C \text {, }
$$

which was studied by S. Takahashi and W. Takahashi [2]. If $\varphi=0$ and $F=0$, then the mixed equilibrium problem (1.1) becomes the following equilibrium problem:

$$
\text { Find } u \in C \text { such that } \Theta(u, y) \geq 0, \quad \forall y \in C \text {. }
$$

If $\Theta(x, y)=0$ for all $x, y \in C$, the mixed equilibrium problem (1.1) becomes the following variational inequality problem:

$$
\text { Find } u \in C \text { such that } \varphi(y)-\varphi(u)+\langle F u, y-u\rangle \geq 0, \quad \forall y \in C \text {. }
$$

The mixed equilibrium problems include fixed point problems, optimization problems, variational inequality problems, Nash equilibrium problems, and the equilibrium problems as special cases; see, for example, [3-8]. Some methods have been proposed to solve the mixed equilibrium problem and the equilibrium problem. In 1997, Flaim and Antipen [4] introduced an iterative method of finding the best approximation to the initial data and proved a strong convergence theorem. Subsequently, S. Takahashi and W. Takahashi [9] introduced another iterative scheme for finding a common element of the set of solutions of the equilibrium problem (1.2) and the set of fixed point points of a nonexpansive mapping. Furthermore, Yao et al. [10] introduced some new iterative schemes for finding a common element of the set of solutions of the equilibrium problem (1.2) and the set of common fixed points of finitely (infinitely) nonexpansive mappings. Very recently, Ceng and Yao [1] considered a new iterative scheme for finding a common element of the set of solutions of the mixed equilibrium problem and the set of common fixed points of finitely many nonexpansive mappings. Peng and Yao [11] developed a CQ method. They obtained some strong convergence results for finding a common element of the set of solutions of the mixed equilibrium problem (1.1) and the set of the variational inequality and the set of fixed points of a nonexpansive mapping. Their results extend and improve the corresponding results in $[1,9,12]$.

Recall that a mapping $B: C \rightarrow C$ is said to be $\beta$-inverse strongly monotone if there exists a constant $\beta>0$ such that $\langle B x-B y, x-y\rangle \geq \beta\|B x-B y\|^{2}$, for all $x, y \in C$. A mapping $A$ is strongly positive on $H$ if there exists a constant $\mu>0$ such that $\langle A x, x\rangle \geq \mu\|x\|^{2}$ for all $x \in H$.

Let $B: H \rightarrow H$ be a single-valued nonlinear mapping and let $R: H \rightarrow 2^{H}$ be a set-valued mapping. Now we concern the following variational inclusion, which is to find a point $x \in H$ such that

$$
\theta \in B(x)+R(x)
$$

where $\theta$ is the zero vector in $H$. The set of solutions of problem (1.6) is denoted by $I(B, R)$. If $H=R^{m}$, then problem (1.6) becomes the generalized equation introduced by Robinson [13]. If $B=0$, then problem (1.6) becomes the inclusion problem introduced by Rockafellar [14]. It is known that (1.6) provides a convenient framework for the unified 
study of optimal solutions in many optimization related areas including mathematical programming, complementarity, variational inequalities, optimal control, mathematical economics, equilibria, and game theory. Also various types of variational inclusions problems have been extended and generalized. Recently, Zhang et al. [15] introduced a new iterative scheme for finding a common element of the set of solutions to the problem (1.6) and the set of fixed points of nonexpansive mappings in Hilbert spaces. Peng et al. [16] introduced another iterative scheme by the viscosity approximate method for finding a common element of the set of solutions of a variational inclusion with set-valued maximal monotone mapping and inverse strongly monotone mappings, the set of solutions of an equilibrium problem, and the set of fixed points of a nonexpansive mapping. For some related works, please see $[1,2,9-11,13-34]$ and the references therein.

Inspired and motivated by the works in the literature, in this paper, we present an iterative algorithm for finding a common element $x^{*}$ of the set of solutions of a mixed equilibrium problem and the set of a variational inclusion in a real Hilbert space. Furthermore, we prove that the proposed iterative algorithms strongly converge to $x^{*}$ which solves some variational inequality.

\section{Preliminaries}

Let $H$ be a real Hilbert space with inner product $\langle\cdot, \cdot\rangle$ and norm $\|\cdot\|$. Let $C$ be a nonempty closed convex subset of $H$. Then, for any $x \in H$, there exists a unique nearest point in $C$, denoted by $P_{C}(x)$, such that

$$
\left\|x-P_{C}(x)\right\| \leq\|x-y\|, \quad \forall y \in C
$$

Such a $P_{C}$ is called the metric projection of $H$ onto $C$. We know that $P_{C}$ is nonexpansive. Further, for $x \in H$ and $x^{*} \in C$,

$$
x^{*}=P_{C}(x) \Longleftrightarrow\left\langle x-x^{*}, x^{*}-y\right\rangle \geq 0, \quad \forall y \in C
$$

A set-valued mapping $T: H \rightarrow 2^{H}$ is called monotone if, for all $x, y \in H, f \in T x$ and $g \in T y$ imply $\langle x-y, f-g\rangle \geq 0$. A monotone mapping $T: H \rightarrow 2^{H}$ is maximal if its graph $G(T)$ is not properly contained in the graph of any other monotone mapping. It is known that a monotone mapping $T$ is maximal if and only if, for $(x, f) \in H \times H,\langle x-y, f-g\rangle \geq 0$ for every $(y, g) \in G(T)$ implies $f \in T x$.

Let the set-valued mapping $R: H \rightarrow 2^{H}$ be maximal monotone. We define the resolvent operator $J_{R, \lambda}$ associated with $R$ and $\lambda$ as follows:

$$
J_{R, \lambda}=(I+\lambda R)^{-1}(x), \quad x \in H,
$$

where $\lambda$ is a positive number. It is worth mentioning that the resolvent operator $J_{R, \lambda}$ is singlevalued, nonexpansive, and 1-inverse strongly monotone and that a solution of problem (1.6) is a fixed point of the operator $J_{R, \lambda}(I-\lambda B)$ for all $\lambda>0$, see, for instance, [25].

Throughout this paper, we assume that a bifunction $\Theta: C \times C \rightarrow \mathbf{R}$ and a convex function $\varphi: C \rightarrow R$ satisfy the following conditions: 
(H1) $\Theta(x, x)=0$ for all $x \in C$;

(H2) $\Theta$ is monotone, that is, $\Theta(x, y)+\Theta(y, x) \leq 0$ for all $x, y \in C$;

(H3) for each $y \in C, x \mapsto \Theta(x, y)$ is weakly upper semicontinuous;

(H4) for each $x \in C, y \mapsto \Theta(x, y)$ is convex and lower semicontinuous;

(H5) for each $x \in C$ and $r>0$, there exists a bounded subset $D_{x} \subset C$ and $y_{x} \in C$ such that for any $z \in C \backslash D_{x}$,

$$
\Theta\left(z, y_{x}\right)+\varphi\left(y_{x}\right)-\varphi(z)+\frac{1}{r}\left\langle y_{x}-z, z-x\right\rangle<0
$$

Lemma 2.1 (see [11]). Let $C$ be a nonempty closed convex subset of a real Hilbert space $H$. Let $\Theta: C \times C \rightarrow R$ be a bifunction and let $\varphi: C \rightarrow R$ be a proper lower semicontinuous and convex function. For $r>0$ and $x \in C$, define a mapping $S_{r}: C \rightarrow C$ as follows:

$$
S_{r}(x)=\left\{z \in C: \Theta(z, y)+\varphi(y)-\varphi(z)+\frac{1}{r}\langle y-z, z-x\rangle \geq 0, \forall y \in C\right\}
$$

for all $x \in C$. Assume that the conditions (H1)-(H5) hold. Then one has the following results:

(1) for each $x \in C, S_{r}(x) \neq \emptyset$ and $S_{r}$ is single-valued;

(2) $S_{r}$ is firmly nonexpansive, that is, for any $x, y \in C$,

$$
\left\|S_{r} x-S_{r} y\right\|^{2} \leq\left\langle S_{r} x-S_{r} y, x-y\right\rangle
$$

(3) $\operatorname{Fix}\left(S_{r}\right)=\mathrm{EP}$;

(4) $\mathrm{EP}$ is closed and convex.

Lemma 2.2 (see [24]). Let $R: H \rightarrow 2^{H}$ be a maximal monotone mapping and let $B: H \rightarrow H$ be a Lipschitz-continuous mapping. Then the mapping $(R+B): H \rightarrow 2^{H}$ is maximal monotone.

Lemma 2.3 (see [34]). Assume taht $\left\{a_{n}\right\}$ is a sequence of nonnegative real numbers such that $a_{n+1} \leq$ $\left(1-\gamma_{n}\right) a_{n}+\delta_{n}$ where $\left\{\gamma_{n}\right\}$ is a sequence in $(0,1)$ and $\left\{\delta_{n}\right\}$ is a sequence such that

(1) $\sum_{n=1}^{\infty} \gamma_{n}=\infty$;

(2) $\lim \sup _{n \rightarrow \infty} \delta_{n} / \gamma_{n} \leq 0$ or $\sum_{n=1}^{\infty}\left|\delta_{n}\right|<\infty$.

Then $\lim _{n \rightarrow \infty} a_{n}=0$.

\section{Main Results}

In this section, we will prove our main result. First, we give some assumptions on the operators and the parameters. Subsequently, we introduce our iterative algorithm for finding a common element of the set of solutions of a mixed equilibrium problem and the set of a variational inclusion. Finally, we will show that the proposed algorithm has strong convergence. 
Let $C$ be a nonempty closed convex subset of a real Hilbert space $H$. Let $\varphi: C \rightarrow R$ be a lower semicontinuous and convex function and let $\Theta: H \times C \rightarrow R$ be a bifunction satisfying conditions (H1)-(H5). Let $A$ be a strongly positive bounded linear operator with coefficient $0<\mu<1$ and let $R: H \rightarrow 2^{H}$ be a maximal monotone mapping. Let the mappings $F, B: C \rightarrow C$ be $\alpha$-inverse strongly monotone and $\beta$-inverse strongly monotone, respectively. Let $r>0$ and $\lambda>0$ be two constants such that $r<2 \alpha$ and $\lambda<2 \beta$.

Now we introduce the following iteration algorithm.

Algorithm 3.1. For given $x_{0} \in C$ arbitrarily, compute the sequences $\left\{x_{n}\right\}$ and $\left\{u_{n}\right\}$ as follows:

$$
\begin{gathered}
\Theta\left(u_{n}, y\right)+\varphi(y)-\varphi\left(u_{n}\right)+\frac{1}{r}\left\langle y-u_{n}, u_{n}-\left(x_{n}-r F x_{n}\right)\right\rangle \geq 0, \quad \forall y \in C, \\
x_{n+1}=P_{C}\left[\left(I-\alpha_{n} A\right) J_{R, \lambda}(I-\lambda B) u_{n}\right],
\end{gathered}
$$

where $\left\{\alpha_{n}\right\}$ is a real sequence in $[0,1]$.

Now we study the strong convergence of the algorithm (3.1).

Theorem 3.2. Suppose that $\Omega:=\mathrm{EP} \cap I(B, R) \neq \emptyset$. Assume the following conditions are satisfied:

(i) $\lim _{n \rightarrow \infty} \alpha_{n}=0$;

(ii) $\sum_{n=0}^{\infty} \alpha_{n}=\infty$;

(iii) $\lim _{n \rightarrow \infty}\left(\alpha_{n+1} / \alpha_{n}\right)=1$.

Then the sequence $\left\{x_{n}\right\}$ generated by (3.1) converges strongly to $x^{*} \in \Omega$ which solves the following variational inequality:

$$
\langle A x, y-x\rangle \geq 0, \quad \forall y \in \Omega .
$$

Proof. Take $x^{*} \in \Omega$. It is clear that

$$
S_{r}\left(x^{*}-r F x^{*}\right)=J_{R, \lambda}\left(x^{*}-\lambda B x^{*}\right)=x^{*}, \quad n \geq 0 .
$$

We divide our proofs into the following five steps:

(1) the sequences $\left\{x_{n}\right\}$ and $\left\{u_{n}\right\}$ are bounded;

(2) $\left\|x_{n+1}-x_{n}\right\| \rightarrow 0$;

(3) $\left\|F x_{n}-F x^{*}\right\| \rightarrow 0$ and $\left\|B u_{n}-B x^{*}\right\| \rightarrow 0$;

(4) $\limsup _{n \rightarrow \infty}\left\langle A x^{*}, x_{n}-x^{*}\right\rangle \geq 0$;

(5) $x_{n} \rightarrow x^{*}$.

Proof of (1). Since $F$ is $\alpha$-inverse strongly monotone and $B$ is $\beta$-inverse strongly monotone, we have

$$
\begin{gathered}
\|(I-r F) x-(I-r F) y\|^{2} \leq\|x-y\|^{2}+r(r-2 \alpha)\|F x-F y\|^{2}, \\
\|(I-\lambda B) x-(I-\lambda B) y\|^{2} \leq\|x-y\|^{2}+\lambda(\lambda-2 \beta)\|B x-B y\|^{2} .
\end{gathered}
$$


It is clear that if $0 \leq r \leq 2 \alpha$ and $0 \leq \lambda \leq 2 \beta$, then $(I-r F)$ and $(I-\lambda B)$ are all nonexpansive. Set $y_{n}=J_{R, \lambda}\left(u_{n}-\lambda B u_{n}\right), n \geq 0$. It follows that

$$
\left\|y_{n}-x^{*}\right\|=\left\|J_{R, \lambda}\left(u_{n}-\lambda B u_{n}\right)-J_{R, \lambda}\left(x^{*}-\lambda B x^{*}\right)\right\| \leq\left\|\left(u_{n}-\lambda B u_{n}\right)-\left(x^{*}-\lambda B x^{*}\right)\right\| \leq\left\|u_{n}-x^{*}\right\| .
$$

By Lemma 2.1, we have $u_{n}=S_{r}\left(x_{n}-r F x_{n}\right)$ for all $n \geq 0$. Then, we have

$$
\begin{aligned}
\left\|u_{n}-x^{*}\right\|^{2} & =\left\|S_{r}\left(x_{n}-r F x_{n}\right)-S_{r}\left(x^{*}-r F x^{*}\right)\right\|^{2} \\
& \leq\left\|x_{n}-r F x_{n}-\left(x^{*}-r F x^{*}\right)\right\|^{2} \\
& \leq\left\|x_{n}-x^{*}\right\|^{2}+r(r-2 \alpha)\left\|F x_{n}-F x^{*}\right\|^{2} \\
& \leq\left\|x_{n}-x^{*}\right\|^{2} .
\end{aligned}
$$

Hence, we have

$$
\left\|y_{n}-x^{*}\right\| \leq\left\|x_{n}-x^{*}\right\|
$$

Since $A$ is linear bounded self-adjoint operator on $H$, then

$$
\|A\|=\sup \{|\langle A u, u\rangle|: u \in H,\|u\|=1\} .
$$

Observe that

$$
\left\langle\left(I-\alpha_{n} A\right) u, u\right\rangle=1-\alpha_{n}\langle A u, u\rangle \geq 1-\alpha_{n}\|A\| \geq 0,
$$

that is to say $I-\alpha_{n} A$ is positive. It follows that

$$
\begin{aligned}
\left\|\left(I-\alpha_{n} A\right)\right\| & =\sup \left\{\left\langle\left(I-\alpha_{n} A\right) u, u\right\rangle: u \in H,\|u\|=1\right\} \\
& =\sup \left\{1-\alpha_{n}\langle A u, u\rangle: u \in H,\|u\|=1\right\} \\
& \leq 1-\alpha_{n} \mu .
\end{aligned}
$$

From (3.1), we deduce that

$$
\begin{aligned}
\left\|x_{n+1}-x^{*}\right\| & =\left\|P_{C}\left[\left(I-\alpha_{n} A\right) y_{n}\right]-x^{*}\right\| \\
& \leq\left\|\left[\left(I-\alpha_{n} A\right)\left(y_{n}-x^{*}\right)\right]-\alpha_{n} A x^{*}\right\| \\
& \leq\left(1-\alpha_{n} \mu\right)\left\|y_{n}-x^{*}\right\|+\alpha_{n}\left\|A x^{*}\right\| \\
& \leq\left(1-\alpha_{n} \mu\right)\left\|x_{n}-x^{*}\right\|+\alpha_{n}\left\|A x^{*}\right\| \\
& \leq \max \left\{\left\|x_{0}-x^{*}\right\|, \frac{\left\|A x^{*}\right\|}{\mu}\right\} .
\end{aligned}
$$

Therefore, $\left\{x_{n}\right\}$ is bounded. Hence, $\left\{u_{n}\right\},\left\{y_{n}\right\}$, and $\left\{A y_{n}\right\}$ are all bounded. 
Proof of (2). From (3.1), we have

$$
\begin{aligned}
\left\|x_{n+1}-x_{n}\right\| & =\left\|P_{C}\left[\left(I-\alpha_{n} A\right) y_{n}\right]-P_{C}\left[\left(I-\alpha_{n-1} A\right) y_{n-1}\right]\right\| \\
& \leq\left\|\left[\left(I-\alpha_{n} A\right) y_{n}\right]-\left[\left(I-\alpha_{n-1} A\right) y_{n-1}\right]\right\| \\
& =\left\|\left(I-\alpha_{n} A\right)\left(y_{n}-y_{n-1}\right)+\left(\alpha_{n-1}-\alpha_{n}\right) A y_{n-1}\right\| \\
& \leq\left\|\left(I-\alpha_{n} A\right)\left(y_{n}-y_{n-1}\right)\right\|+\left\|\left(\alpha_{n-1}-\alpha_{n}\right) A y_{n-1}\right\| \\
& \leq\left(1-\alpha_{n} \mu\right)\left\|y_{n}-y_{n-1}\right\|+\left|\alpha_{n}-\alpha_{n-1}\right|\left\|A y_{n-1}\right\| .
\end{aligned}
$$

Note that

$$
\begin{aligned}
\left\|y_{n}-y_{n-1}\right\| & =\left\|J_{R, \lambda}\left(u_{n}-\lambda B u_{n}\right)-J_{R, \lambda}\left(u_{n-1}-\lambda B u_{n-1}\right)\right\| \\
& \leq\left\|\left(u_{n}-\lambda B u_{n}\right)-\left(u_{n-1}-\lambda B u_{n-1}\right)\right\| \\
& \leq\left\|u_{n}-u_{n-1}\right\| \\
& =\left\|S_{r}\left(x_{n}-r F x_{n}\right)-S_{r}\left(x_{n-1}-r F x_{n-1}\right)\right\| \\
& \leq\left\|\left(x_{n}-r F x_{n}\right)-\left(x_{n-1}-r F x_{n-1}\right)\right\| \\
& \leq\left\|x_{n}-x_{n-1}\right\| .
\end{aligned}
$$

Substituting (3.14) into (3.13), we get

$$
\begin{aligned}
\left\|x_{n+1}-x_{n}\right\| & \leq\left(1-\alpha_{n} \mu\right)\left\|x_{n}-x_{n-1}\right\|+\left|\alpha_{n}-\alpha_{n-1}\right|\left\|A y_{n-1}\right\| \\
& =\left(1-\alpha_{n} \mu\right)\left\|x_{n}-x_{n-1}\right\|+\alpha_{n} \mu\left|1-\frac{\alpha_{n-1}}{\alpha_{n}}\right| \frac{1}{\mu}\left\|A y_{n-1}\right\| .
\end{aligned}
$$

Notice that $\lim _{n \rightarrow \infty}\left|1-\alpha_{n-1} / \alpha_{n}\right|=0$. This together with the last inequality and Lemma 2.3 implies that

$$
\lim _{n \rightarrow \infty}\left\|x_{n+1}-x_{n}\right\|=0
$$

Proof of (3). From (3.5) and (3.7), we get

$$
\begin{aligned}
\left\|y_{n}-x^{*}\right\|^{2} & =\left\|J_{R, \lambda}\left(u_{n}-\lambda B u_{n}\right)-J_{R, \lambda}\left(x^{*}-\lambda B x^{*}\right)\right\|^{2} \\
& \leq\left\|\left(u_{n}-\lambda B u_{n}\right)-\left(x^{*}-\lambda B x^{*}\right)\right\|^{2} \\
& \leq\left\|u_{n}-x^{*}\right\|^{2}+\lambda(\lambda-2 \beta)\left\|B u_{n}-B x^{*}\right\|^{2} \\
& \leq\left\|x_{n}-x^{*}\right\|^{2}+r(r-2 \alpha)\left\|F x_{n}-F x^{*}\right\|^{2}+\lambda(\lambda-2 \beta)\left\|B u_{n}-B x^{*}\right\|^{2} .
\end{aligned}
$$


By (3.1), we obtain

$$
\begin{aligned}
\left\|x_{n+1}-x^{*}\right\|^{2} & =\left\|P_{C}\left[\left(I-\alpha_{n} A\right) y_{n}\right]-x^{*}\right\|^{2} \\
& \leq\left\|\left(I-\alpha_{n} A\right) y_{n}-x^{*}\right\|^{2} \\
& =\left\|y_{n}-x^{*}-\alpha_{n} A y_{n}\right\|^{2} \\
& =\left\|y_{n}-x^{*}\right\|^{2}-2 \alpha_{n}\left\langle y_{n}-x^{*}, A y_{n}\right\rangle+\alpha_{n}^{2}\left\|A y_{n}\right\|^{2} \\
& \leq\left\|y_{n}-x^{*}\right\|^{2}+\alpha_{n}\left(2\left\|y_{n}-x^{*}\right\|\left\|A y_{n}\right\|+\left\|A y_{n}\right\|^{2}\right) \\
& \leq\left\|y_{n}-x^{*}\right\|^{2}+\alpha_{n} M,
\end{aligned}
$$

where $M>0$ is some constant satisfying $\sup _{n}\left\{2\left\|y_{n}-x^{*}\right\|\left\|A y_{n}\right\|+\left\|A y_{n}\right\|^{2}\right\} \leq M$. From (3.17) and (3.18), we have

$$
\begin{aligned}
\left\|x_{n+1}-x^{*}\right\|^{2} \leq & \left\|x_{n}-x^{*}\right\|^{2}+r(r-2 \alpha)\left\|F x_{n}-F x^{*}\right\|^{2} \\
& +\lambda(\lambda-2 \beta)\left\|B u_{n}-B x^{*}\right\|^{2}+\alpha_{n} M .
\end{aligned}
$$

Thus,

$$
\begin{aligned}
& r(2 \alpha-r)\left\|F x_{n}-F x^{*}\right\|^{2}+\lambda(2 \beta-\lambda)\left\|B u_{n}-B x^{*}\right\|^{2} \\
& \quad \leq\left\|x_{n}-x^{*}\right\|^{2}-\left\|x_{n+1}-x^{*}\right\|^{2}+\alpha_{n} M \\
& \quad \leq\left(\left\|x_{n}-x^{*}\right\|+\left\|x_{n+1}-x^{*}\right\|\right)\left\|x_{n+1}-x_{n}\right\|+\alpha_{n} M,
\end{aligned}
$$

which imply that

$$
\lim _{n \rightarrow \infty}\left\|F x_{n}-F x^{*}\right\|=0, \quad \lim _{n \rightarrow \infty}\left\|B u_{n}-B x^{*}\right\|=0 .
$$


Proof of (4). Since $S_{r}$ is firmly nonexpansive, we have

$$
\begin{aligned}
\left\|u_{n}-x^{*}\right\|^{2}= & \left\|S_{r}\left(x_{n}-r F x_{n}\right)-S_{r}\left(x^{*}-r F x^{*}\right)\right\|^{2} \\
\leq & \left\langle x_{n}-r F x_{n}-\left(x^{*}-r F x^{*}\right), u_{n}-x^{*}\right\rangle \\
= & \frac{1}{2}\left(\left\|x_{n}-r F x_{n}-\left(x^{*}-r F x^{*}\right)\right\|^{2}+\left\|u_{n}-x^{*}\right\|^{2}\right. \\
& \left.\quad-\left\|x_{n}-r F x_{n}-\left(x^{*}-r F x^{*}\right)-\left(u_{n}-x^{*}\right)\right\|^{2}\right) \\
\leq & \frac{1}{2}\left(\left\|x_{n}-x^{*}\right\|^{2}+\left\|u_{n}-x^{*}\right\|^{2}-\left\|x_{n}-u_{n}-r\left(F x_{n}-F x^{*}\right)\right\|^{2}\right) \\
= & \frac{1}{2}\left(\left\|x_{n}-x^{*}\right\|^{2}+\left\|u_{n}-x^{*}\right\|^{2}-\left\|x_{n}-u_{n}\right\|^{2}\right. \\
& \left.\quad+2 r\left\langle F x_{n}-F x^{*}, x_{n}-u_{n}\right\rangle-r^{2}\left\|F x_{n}-F x^{*}\right\|^{2}\right) .
\end{aligned}
$$

Hence, we have

$$
\left\|u_{n}-x^{*}\right\|^{2} \leq\left\|x_{n}-x^{*}\right\|^{2}-\left\|x_{n}-u_{n}\right\|^{2}+2 r\left\|F x_{n}-F x^{*}\right\|\left\|x_{n}-u_{n}\right\| .
$$

Since $J_{R, \lambda}$ is 1 -inverse strongly monotone, we have

$$
\begin{aligned}
\left\|y_{n}-x^{*}\right\|^{2}= & \left\|J_{R, \lambda}\left(u_{n}-\lambda B u_{n}\right)-J_{R, \lambda}\left(x^{*}-\lambda B x^{*}\right)\right\|^{2} \\
\leq & \left\langle u_{n}-\lambda B u_{n}-\left(x^{*}-\lambda B x^{*}\right), y_{n}-x^{*}\right\rangle \\
= & \frac{1}{2}\left(\left\|u_{n}-\lambda B u_{n}-\left(x^{*}-\lambda B x^{*}\right)\right\|^{2}+\left\|y_{n}-x^{*}\right\|^{2}\right. \\
& \left.\quad-\left\|u_{n}-\lambda B u_{n}-\left(x^{*}-\lambda B x^{*}\right)-\left(y_{n}-x^{*}\right)\right\|^{2}\right) \\
\leq & \frac{1}{2}\left(\left\|u_{n}-x^{*}\right\|^{2}+\left\|y_{n}-x^{*}\right\|^{2}-\left\|u_{n}-y_{n}-\lambda\left(B u_{n}-B x^{*}\right)\right\|^{2}\right) \\
= & \frac{1}{2}\left(\left\|u_{n}-x^{*}\right\|^{2}+\left\|y_{n}-x^{*}\right\|^{2}-\left\|u_{n}-y_{n}\right\|^{2}\right. \\
& \left.\quad+2 \lambda\left\langle B u_{n}-B x^{*}, u_{n}-y_{n}\right\rangle-\lambda^{2}\left\|B u_{n}-B x^{*}\right\|^{2}\right),
\end{aligned}
$$

which implies that

$$
\left\|y_{n}-x^{*}\right\|^{2} \leq\left\|u_{n}-x^{*}\right\|^{2}-\left\|u_{n}-y_{n}\right\|^{2}+2 \lambda\left\|B u_{n}-B x^{*}\right\|\left\|u_{n}-y_{n}\right\|
$$


Thus, by (3.23) and (3.25), we obtain

$$
\begin{aligned}
\left\|y_{n}-x^{*}\right\|^{2} \leq & \left\|x_{n}-x^{*}\right\|^{2}-\left\|x_{n}-u_{n}\right\|^{2}+2 r\left\|F x_{n}-F x^{*}\right\|\left\|x_{n}-u_{n}\right\| \\
& -\left\|u_{n}-y_{n}\right\|^{2}+2 \lambda\left\|B u_{n}-B x^{*}\right\|\left\|u_{n}-y_{n}\right\| .
\end{aligned}
$$

Substitute (3.26) into (3.18) to get

$$
\begin{aligned}
\left\|x_{n+1}-x^{*}\right\|^{2} \leq & \left\|x_{n}-x^{*}\right\|^{2}-\left\|x_{n}-u_{n}\right\|^{2}+2 r\left\|F x_{n}-F x^{*}\right\|\left\|x_{n}-u_{n}\right\| \\
& -\left\|u_{n}-y_{n}\right\|^{2}+2 \lambda\left\|B u_{n}-B x^{*}\right\|\left\|u_{n}-y_{n}\right\|+\alpha_{n} M .
\end{aligned}
$$

Then we derive

$$
\begin{aligned}
\| x_{n}- & u_{n}\left\|^{2}+\right\| u_{n}-y_{n} \|^{2} \\
\leq & \left(\left\|x_{n}-x^{*}\right\|+\left\|x_{n+1}-x^{*}\right\|\right)\left\|x_{n+1}-x_{n}\right\|+2 r\left\|F x_{n}-F x^{*}\right\|\left\|x_{n}-u_{n}\right\| \\
& +2 \lambda\left\|B u_{n}-B x^{*}\right\|\left\|u_{n}-y_{n}\right\|+\alpha_{n} M .
\end{aligned}
$$

So, we have

$$
\lim _{n \rightarrow \infty}\left\|x_{n}-u_{n}\right\|=0, \quad \lim _{n \rightarrow \infty}\left\|u_{n}-y_{n}\right\|=0
$$

Proof of (5). We note that $P_{\Omega}(I-A)$ is a contraction. As a matter of fact,

$$
\begin{aligned}
\left\|P_{\Omega}(I-A) x-P_{\Omega}(I-A) y\right\| & \leq\left\|(I-A) x-P_{\Omega}(I-A) y\right\| \\
& \leq\|I-A\|\|x-y\| \\
& \leq(1-\mu)\|x-y\|
\end{aligned}
$$

for all $x, y \in H$. Hence $P_{\Omega}(I-A)$ has a unique fixed point, say $x^{*} \in \Omega$. That is, $x^{*}=P_{\Omega}(I-$ $A)\left(x^{*}\right)$. This implies that $\left\langle A x^{*}, y-x^{*}\right\rangle \geq 0$ for all $y \in \Omega$. Next, we prove that

$$
\limsup _{n \rightarrow \infty}\left\langle A x^{*}, x_{n}-x^{*}\right\rangle \geq 0
$$

First, we note that there exists a subsequence $\left\{x_{n_{i}}\right\}$ of $\left\{x_{n}\right\}$ such that

$$
\limsup _{n \rightarrow \infty}\left\langle A x^{*}, x_{n}-x^{*}\right\rangle=\lim _{j \rightarrow \infty}\left\langle A x^{*}, x_{n_{j}}-x^{*}\right\rangle .
$$

Since $\left\{x_{n_{j}}\right\}$ is bounded, there exists a subsequence $\left\{x_{n_{j i}}\right\}$ of $\left\{x_{n_{j}}\right\}$ which converges weakly to $w$. Without loss of generality, we can assume that $x_{n_{j}} \rightarrow w$. 
Fixed Point Theory and Applications

We next show that $w \in$ EP. By $u_{n}=S_{r}\left(x_{n}-r F x_{n}\right)$, we know that

$$
\Theta\left(u_{n}, y\right)+\varphi(y)-\varphi\left(u_{n}\right)+\frac{1}{r}\left\langle y-u_{n}, u_{n}-\left(x_{n}-r F x_{n}\right)\right\rangle \geq 0, \quad \forall y \in C
$$

It follows from $(\mathrm{H} 2)$ that

$$
\varphi(y)-\varphi\left(u_{n}\right)+\frac{1}{r}\left\langle y-u_{n}, u_{n}-\left(x_{n}-r F x_{n}\right)\right\rangle \geq \Theta\left(y, u_{n}\right), \quad \forall y \in C .
$$

Hence,

$$
\varphi(y)-\varphi\left(u_{n_{i}}\right)+\left\langle y-u_{n_{i}}, \frac{u_{n_{i}}-\left(x_{n_{i}}-r F x_{n_{i}}\right)}{r}\right\rangle \geq \Theta\left(y, u_{n_{i}}\right), \quad \forall y \in C .
$$

For $t \in(0,1]$ and $y \in H$, let $y_{t}=t y+(1-t) w$. From (3.35) we have

$$
\begin{aligned}
\left\langle y_{t}-u_{n_{i}}, F y_{t}\right\rangle \geq & \left\langle y_{t}-u_{n_{i}}, F y_{t}\right\rangle-\varphi\left(y_{t}\right)+\varphi\left(u_{n_{i}}\right) \\
& -\left\langle y_{t}-u_{n_{i}}, \frac{u_{n_{i}}-\left(x_{n_{i}}-r F x_{n_{i}}\right)}{r}\right\rangle+\Theta\left(y_{t}, u_{n_{i}}\right) \\
= & \left\langle y_{t}-u_{n_{i}}, F y_{t}-F u_{n_{i}}\right\rangle+\left\langle y_{t}-u_{n_{i}}, F u_{n_{i}}-F x_{n_{i}}\right\rangle-\varphi\left(y_{t}\right) \\
& +\varphi\left(u_{n_{i}}\right)-\left\langle y_{t}-u_{n_{i}}, \frac{u_{n_{i}}-x_{n_{i}}}{r}\right\rangle+\Theta\left(y_{t}, u_{n_{i}}\right) .
\end{aligned}
$$

Since $\left\|u_{n_{i}}-x_{n_{i}}\right\| \rightarrow 0$, we have $\left\|F u_{n_{i}}-F x_{n_{i}}\right\| \rightarrow 0$. Further, from the inverse strongly monotonicity of $F$, we have $\left\langle y_{t}-u_{n_{i}}, F y_{t}-F u_{n_{i}}\right\rangle \geq 0$. So, from (H4) and the weakly lower semicontinuity of $\varphi,\left(u_{n_{i}}-x_{n_{i}}\right) / r \rightarrow 0$ and $u_{n_{i}} \rightarrow w$ weakly, we have

$$
\left\langle y_{t}-w, F y_{t}\right\rangle \geq-\varphi\left(y_{t}\right)+\varphi(w)+\Theta\left(y_{t}, w\right) .
$$

From (H1), (H4), and (3.37), we also have

$$
\begin{aligned}
0 & =\Theta\left(y_{t}, y_{t}\right)+\varphi\left(y_{t}\right)-\varphi\left(y_{t}\right) \\
& \leq t \Theta\left(y_{t}, y\right)+(1-t) \Theta\left(y_{t}, w\right)+t \varphi(y)+(1-t) \varphi(w)-\varphi\left(y_{t}\right) \\
& =t\left[\Theta\left(y_{t}, y\right)+\varphi(y)-\varphi\left(y_{t}\right)\right]+(1-t)\left[\Theta\left(y_{t}, w\right)+\varphi(w)-\varphi\left(y_{t}\right)\right] \\
& \leq t\left[\Theta\left(y_{t}, y\right)+\varphi(y)-\varphi\left(y_{t}\right)\right]+(1-t)\left\langle y_{t}-w, F y_{t}\right\rangle \\
& =t\left[\Theta\left(y_{t}, y\right)+\varphi(y)-\varphi\left(y_{t}\right)\right]+(1-t) t\left\langle y-w, F y_{t}\right\rangle,
\end{aligned}
$$

and hence

$$
0 \leq \Theta\left(y_{t}, y\right)+\varphi(y)-\varphi\left(y_{t}\right)+(1-t)\left\langle y-w, F y_{t}\right\rangle
$$


Letting $t \rightarrow 0$, we have, for each $y \in C$,

$$
\Theta(w, y)+\varphi(y)-\varphi(w)+\langle y-w, F w\rangle \geq 0
$$

This implies that $w \in$ EP.

Next, we show that $w \in I(B, R)$. In fact, since $B$ is $\beta$-inverse strongly monotone, $B$ is Lipschitz continuous monotone mapping. It follows from Lemma 2.2 that $R+B$ is maximal monotone. Let $(v, g) \in G(R+B)$, that is, $g-B v \in R(v)$. Again since $y_{n_{i}}=J_{R, \lambda}\left(u_{n_{i}}-\lambda B u_{n-i}\right)$, we have $u_{n_{i}}-\lambda u_{n_{i}} \in(I+\lambda R)\left(y_{n_{i}}\right)$, that is, $(1 / \lambda)\left(u_{n_{i}}-y_{n_{i}}-\lambda B u_{n_{i}}\right) \in R\left(y_{n_{i}}\right)$. By virtue of the maximal monotonicity of $R+B$, we have

$$
\left\langle v-y_{n_{i}}, g-B v-\frac{1}{\lambda}\left(u_{n_{i}}-y_{n_{i}}-\lambda B u_{n_{i}}\right)\right\rangle \geq 0
$$

and so

$$
\begin{aligned}
\left\langle v-y_{n_{i}}, g\right\rangle & \geq\left\langle v-y_{n_{i}}, B v+\frac{1}{\lambda}\left(u_{n_{i}}-y_{n_{i}}-\lambda B u_{n_{i}}\right)\right\rangle \\
& =\left\langle v-y_{n_{i}}, B v-B y_{n_{i}}+B y_{n_{i}}-B u_{n_{i}}+\frac{1}{\lambda}\left(u_{n_{i}}-y_{n_{i}}\right)\right\rangle \\
& \geq\left\langle v-y_{n_{i}}, B y_{n_{i}}-B u_{n_{i}}\right\rangle+\left\langle v-y_{n_{i}}, \frac{1}{\lambda}\left(u_{n_{i}}-y_{n_{i}}\right)\right\rangle .
\end{aligned}
$$

It follows from $\left\|u_{n}-y_{n}\right\| \rightarrow 0,\left\|B u_{n}-B y_{n}\right\| \rightarrow 0$ and $y_{n_{i}} \rightarrow w$ that

$$
\lim _{n_{i} \rightarrow \infty}\left\langle v-y_{n_{i}}, g\right\rangle=\langle v-w, g\rangle \geq 0
$$

It follows from the maximal monotonicity of $B+R$ that $\theta \in(R+B)(w)$, that is, $w \in I(B, R)$. Therefore, $w \in \Omega$. It follows that

$$
\begin{aligned}
\limsup _{n \rightarrow \infty}\left\langle A x^{*}, x_{n}-x^{*}\right\rangle & =\lim _{j \rightarrow \infty}\left\langle A x^{*}, x_{n_{j}}-x^{*}\right\rangle \\
& =\left\langle A x^{*}, w-x^{*}\right\rangle \\
& \geq 0 .
\end{aligned}
$$

Proof of (6). First, we note that $x_{n+1}=P_{C}\left[\left(I-\alpha_{n} A\right) y_{n}\right]$; then for all $x \in C$, we have $\left\langle x_{n+1}-(I-\right.$ $\left.\left.\alpha_{n} A\right) y_{n}, x_{n+1}-x\right\rangle \leq 0$. 
Fixed Point Theory and Applications

From (3.1), we have

$$
\begin{aligned}
\left\|x_{n+1}-x^{*}\right\|^{2}= & \left\langle x_{n+1}-x^{*}, x_{n+1}-x^{*}\right\rangle \\
= & \left\langle x_{n+1}-\left(I-\alpha_{n} A\right) y_{n}+\left(I-\alpha_{n} A\right) y_{n}-x^{*}, x_{n+1}-x^{*}\right\rangle \\
= & \left\langle x_{n+1}-\left(I-\alpha_{n} A\right) y_{n}, x_{n+1}-x^{*}\right\rangle \\
& +\left\langle\left(I-\alpha_{n} A\right) y_{n}-x^{*}, x_{n+1}-x^{*}\right\rangle \\
\leq & \left\langle\left(I-\alpha_{n} A\right)\left(y_{n}-x^{*}\right)-\alpha_{n} A x^{*}, x_{n+1}-x^{*}\right\rangle \\
= & \left\langle\left(I-\alpha_{n} A\right)\left(y_{n}-x^{*}\right), x_{n+1}-x^{*}\right\rangle \\
& +2 \alpha_{n}\left\langle-A x^{*}, x_{n+1}-x^{*}\right\rangle \\
\leq & \left\|\left(I-\alpha_{n} A\right)\left(y_{n}-x^{*}\right)\right\|\left\|x_{n+1}-x^{*}\right\| \\
& +2 \alpha_{n}\left\langle-A x^{*}, x_{n+1}-x^{*}\right\rangle \\
\leq & \frac{\left(1-\alpha_{n} \mu\right)}{2}\left(\left\|x_{n}-x^{*}\right\|^{2}+\left\|x_{n+1}-x^{*}\right\|^{2}\right) \\
& +2 \alpha_{n}\left\langle-A x^{*}, x_{n+1}-x^{*}\right\rangle,
\end{aligned}
$$

that is,

$$
\begin{aligned}
\left\|x_{n+1}-x^{*}\right\|^{2} & \leq\left(1-\alpha_{n} \mu\right)\left\|x_{n}-x^{*}\right\|^{2}+\frac{2 \alpha_{n}}{1+\alpha_{n} \mu}\left\langle-A x^{*}, x_{n+1}-x^{*}\right\rangle \\
& =\left(1-\delta_{n}\right)\left\|x_{n}-x^{*}\right\|^{2}+\delta_{n} \sigma_{n}
\end{aligned}
$$

where $\delta_{n}=\alpha_{n} \mu$ and $\sigma_{n}=\left(2 /\left(1+\alpha_{n} \mu\right) \mu\right)\left\langle-A x^{*}, x_{n+1}-x^{*}\right\rangle$. It is easy to see that $\sum_{n=1}^{\infty} \delta_{n}=\infty$ and $\lim \sup _{n \rightarrow \infty} \sigma_{n} \leq 0$. Hence, by Lemma 2.3, we conclude that the sequence $\left\{x_{n}\right\}$ converges strongly to $x^{*}$. This completes the proof.

\section{Acknowledgment}

The author was supported in part by NSC 98-2622-E-230-006-CC3 and NSC 98-2923-E-110003-MY3.

\section{References}

[1] L.-C. Ceng and J.-C. Yao, "A hybrid iterative scheme for mixed equilibrium problems and fixed point problems," Journal of Computational and Applied Mathematics, vol. 214, no. 1, pp. 186-201, 2008.

[2] S. Takahashi and W. Takahashi, "Strong convergence theorem for a generalized equilibrium problem and a nonexpansive mapping in a Hilbert space," Nonlinear Analysis: Theory, Methods \& Applications, vol. 69, no. 3, pp. 1025-1033, 2008.

[3] E. Blum and W. Oettli, "From optimization and variational inequalities to equilibrium problems," The Mathematics Student, vol. 63, no. 1-4, pp. 123-145, 1994. 
[4] S. D. Flåm and A. S. Antipin, "Equilibrium programming using proximal-like algorithms," Mathematical Programming, vol. 78, no. 1, pp. 29-41, 1997.

[5] L.-C. Zeng, S.-Y. Wu, and J.-C. Yao, “Generalized KKM theorem with applications to generalized minimax inequalities and generalized equilibrium problems," Taiwanese Journal of Mathematics, vol. 10, no. 6, pp. 1497-1514, 2006.

[6] O. Chadli, N. C. Wong, and J. C. Yao, "Equilibrium problems with applications to eigenvalue problems," Journal of Optimization Theory and Applications, vol. 117, no. 2, pp. 245-266, 2003.

[7] O. Chadli, S. Schaible, and J. C. Yao, "Regularized equilibrium problems with application to noncoercive hemivariational inequalities," Journal of Optimization Theory and Applications, vol. 121, no. 3, pp. 571-596, 2004.

[8] I. V. Konnov, S. Schaible, and J. C. Yao, "Combined relaxation method for mixed equilibrium problems," Journal of Optimization Theory and Applications, vol. 126, no. 2, pp. 309-322, 2005.

[9] S. Takahashi and W. Takahashi, "Viscosity approximation methods for equilibrium problems and fixed point problems in Hilbert spaces," Journal of Mathematical Analysis and Applications, vol. 331, no. 1, pp. 506-515, 2007.

[10] Y. Yao, Y.-C. Liou, and J.-C. Yao, "Convergence theorem for equilibrium problems and fixed point problems of infinite family of nonexpansive mappings," Fixed Point Theory and Applications, vol. 2007, Article ID 64363, 12 pages, 2007.

[11] J.-W. Peng and J.-C. Yao, "A new hybrid-extragradient method for generalized mixed equilibrium problems, fixed point problems and variational inequality problems," Taiwanese Journal of Mathematics, vol. 12, no. 6, pp. 1401-1432, 2008.

[12] P. L. Combettes and S. A. Hirstoaga, "Equilibrium programming in Hilbert spaces," Journal of Nonlinear and Convex Analysis, vol. 6, no. 1, pp. 117-136, 2005.

[13] S. M. Robinson, "Generalized equations and their solutions. I. Basic theory," Mathematical Programming Study, no. 10, pp. 128-141, 1979.

[14] R. T. Rockafellar, "Monotone operators and the proximal point algorithm," SIAM Journal on Control and Optimization, vol. 14, no. 5, pp. 877-898, 1976.

[15] S.-S. Zhang, J. H. W. Lee, and C. K. Chan, "Algorithms of common solutions to quasi variational inclusion and fixed point problems," Applied Mathematics and Mechanics. English Edition, vol. 29, no. 5, pp. 571-581, 2008.

[16] J.-W. Peng, Y. Wang, D. S. Shyu, and J.-C. Yao, “Common solutions of an iterative scheme for variational inclusions, equilibrium problems, and fixed point problems," Journal of Inequalities and Applications, vol. 2008, Article ID 720371, 15 pages, 2008.

[17] X. P. Ding, Y. C. Lin, and J. C. Yao, "Predictor-corrector algorithms for solving generalized mixed implicit quasi-equilibrium problems," Applied Mathematics and Mechanics, vol. 27, no. 9, pp. 1157-1164, 2006.

[18] S. Plubtieng and R. Punpaeng, "A general iterative method for equilibrium problems and fixed point problems in Hilbert spaces," Journal of Mathematical Analysis and Applications, vol. 336, no. 1, pp. 455$469,2007$.

[19] A. Tada and W. Takahashi, "Strong convergence theorem for an equilibrium problem and a nonexpansive mapping," in Nonlinear Analysis and Convex Analysis, W. Takahashi and T. Tanaka, Eds., pp. 609-617, Yokohama Publishers, Yokohama, Japan, 2007.

[20] K. Shimoji and W. Takahashi, "Strong convergence to common fixed points of infinite nonexpansive mappings and applications," Taiwanese Journal of Mathematics, vol. 5, no. 2, pp. 387-404, 2001.

[21] Y. Yao and J.-C. Yao, “On modified iterative method for nonexpansive mappings and monotone mappings," Applied Mathematics and Computation, vol. 186, no. 2, pp. 1551-1558, 2007.

[22] A. Moudafi, "Viscosity approximation methods for fixed-points problems," Journal of Mathematical Analysis and Applications, vol. 241, no. 1, pp. 46-55, 2000.

[23] S. Adly, "Perturbed algorithms and sensitivity analysis for a general class of variational inclusions," Journal of Mathematical Analysis and Applications, vol. 201, no. 2, pp. 609-630, 1996.

[24] H. Brézis, Opérateurs Maximaux Monotones et Semi-Groupes de Contractions dans les Espaces de Hilbert, North-Holland Mathematics Studies, no. 5, North-Holland, Amsterdam, The Netherlands, 1973.

[25] B. Lemaire, "Which fixed point does the iteration method select?" in Recent Advances in Optimization (Trier, 1996), vol. 452 of Lecture Notes in Economics and Mathematical Systems, pp. 154-167, Springer, Berlin, Germany, 1997.

[26] Y. Yao and Y.-C. Liou, "Weak and strong convergence of Krasnoselski-Mann iteration for hierarchical fixed point problems," Inverse Problems, vol. 24, no. 1, Article ID 015015, 8 pages, 2008. 
[27] Y. Yao, Y.-C. Liou, and J.-C. Yao, "An iterative algorithm for approximating convex minimization problem," Applied Mathematics and Computation, vol. 188, no. 1, pp. 648-656, 2007.

[28] Y. Yao, R. Chen, and J.-C. Yao, "Strong convergence and certain control conditions for modified Mann iteration," Nonlinear Analysis: Theory, Methods \& Applications, vol. 68, no. 6, pp. 1687-1693, 2008.

[29] X. P. Ding, "Perturbed Ishikawa type iterative algorithm for generalized quasivariational inclusions," Applied Mathematics and Computation, vol. 141, no. 2-3, pp. 359-373, 2003.

[30] N.-J. Huang, "Mann and Ishikawa type perturbed iterative algorithms for generalized nonlinear implicit quasi-variational inclusions," Computers $\mathcal{E}$ Mathematics with Applications, vol. 35, no. 10, pp. $1-7,1998$.

[31] Y.-P. Fang and N.-J. Huang, "H-monotone operator and resolvent operator technique for variational inclusions," Applied Mathematics and Computation, vol. 145, no. 2-3, pp. 795-803, 2003.

[32] L.-J. Lin, "Variational inclusions problems with applications to Ekeland's variational principle, fixed point and optimization problems," Journal of Global Optimization, vol. 39, no. 4, pp. 509-527, 2007.

[33] R. U. Verma, "General system of $(A, \eta)$-monotone variational inclusion problems based on generalized hybrid iterative algorithm," Nonlinear Analysis: Hybrid Systems, vol. 1, no. 3, pp. 326-335, 2007.

[34] H.-K. Xu, "Viscosity approximation methods for nonexpansive mappings," Journal of Mathematical Analysis and Applications, vol. 298, no. 1, pp. 279-291, 2004. 